\title{
Elección de escuela en Chile: De las dinámicas de distinción y exclusión a la segregación socioeconómica del sistema escolar
}

\author{
School choice in Chile: From distinction and exclusion to the social \\ segregation of the school system \\ Escolha de escola no Chile: Das dinâmicas de distinção e exclusão \\ á segregação socioeconômica do sistema educacional \\ Hernández, Macarena ${ }^{a}$; Raczynski, Dagmar ${ }^{b}$

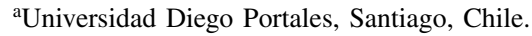 \\ Teléfono: 02-28234196. Correo electrónico: macahernandezvejar@gmail.com \\ ${ }^{\mathrm{b}}$ Asesorías para el Desarrollo, Santiago, Chile. \\ Teléfono: 02-22368199. Correo electrónico: dagmar.raczynski@asesoriasparaeldesarrollo.cl
}

\begin{abstract}
RESUMEN
La literatura internacional ha constatado desde hace más de una década la estrecha vinculación entre elección de escuela, clase social y segregación social de los sistemas educativos. En Chile, en cambio, es solo recientemente que el estudio de los procesos de elección en el marco de la alta segregación y estratificación del sistema escolar ha emergido dentro de la agenda investigativa. Avanzando en esta línea, este artículo profundiza y contrasta las dinámicas de elección de familias de clase media y baja, desde un enfoque cualitativo. Los resultados cuestionan decididamente los principios discursivos sostenidos por los defensores del school choice, aportando evidencia respecto de los procesos mediante los cuales las familias chilenas de ambos estratos fabrican la segregación y estratificación del sistema, a partir de sus lógicas de elección educativa, en el contexto de un diseño institucional de política que acentúa estas lógicas más que neutralizarlas.
\end{abstract}

Palabras clave: elección de escuela, segregación socioeconómica, clases sociales, Chile, política educativa.

\begin{abstract}
International studies have claimed for many decades the relationship between school choice, social class and social segregation in education systems. In Chile, in contrast, the analysis of parental choice processes, in the context of the high level of social segregation within the school system, have emerged only recently within the research agenda. Within these trends, this paper explores and contrasts the dynamics of school choice of middle and lower classes, from a qualitative perspective. The results question the discursive principles held by advocates of school choice, providing evidence about the processes by which families produce and contribute, by their school choices, to the segregation and stratification of the system, in the context of the specific policy design in which school choice is currently enacted.
\end{abstract}

Key words: school choice, social segregation, social class, Chile, education policy.

\section{RESUMO}

A literatura internacional tem constatado, há mais de uma década, a estreita ligação entre escolha de escola, classe social e segregação social dos sistemas educativos. No Chile, no entanto, só recentemente, é que o estudo dos processos de escolha no contexto da alta segregação e estratificação do sistema escolar tem emergido nas investigações científicas. Avançando nessa linha, aprofundam-se e contrastam-se as dinâmicas de escolha de famílias de classe média e baixa, desde uma abordagem qualitativa. Resultados questionam fortemente os princípios discursivos sustentados pelos defensores do school choice, fornecendo evidências sobre os processos por meio dos quais as famílias chilenas de ambos os estratos fabricam a segregação e estratificação do sistema, a partir das suas lógicas de escolha educativa, no contexto de um projeto institucional de política que enfatiza tal lógica em vez de neutralizá-la.

Palavras chave: escolha de escola, segregação socioeconômica, classes sociais, Chile, política educativa. 


\section{INTRODUCCIÓN}

El alarmante nivel de segregación y estratificación del sistema escolar chileno se ha instalado fuertemente dentro de la agenda de investigación y la discusión de política educativa del país. La gran mayoría de los estudios enfocados en revelar los factores tras este fenómeno se han situado desde la perspectiva de la oferta (los establecimientos escolares y sus sostenedores), erigiendo como factores propulsores claves al financiamiento compartido y a los procesos de selección de alumnos por parte de los colegios (Hsieh \& Urquiola, 2006; Bellei, 2007; García-Huidobro, 2007; Valenzuela, 2008; Contreras, Sepúlveda \& Bustos, 2010).

En este escenario, menor atención se ha prestado al conocimiento y a la investigación desde el lado de las demandas o las familias, y sus procesos de elección de escuela. La falta de incorporación de esta mirada sorprende si se toma en cuenta que el sistema de libre school choice se ha instaurado y sostenido por varias décadas en el país como principio fundamental de la política educativa chilena, y más aún si se considera la principal conclusión avanzada por la literatura internacional en el tema que indica que los procesos de elección contribuyen, refuerzan y "fabrican" la segregación socioeconómica de los sistemas educativos (Van Zanten \& Ball, 2002).

Tomando en cuenta estos antecedentes, el estudio de los procesos de elección de las familias resulta ser esencial si se quiere completar y complementar la evidencia disponible respecto de las causas asociadas a la segregación y estratificación del sistema escolar chileno. Ahora bien, a diferencia de evidencia más reciente en esta línea (Gubbins, 2013; Córdoba, 2014) este artículo parte desde el supuesto fundamental de que la comprensión del vínculo entre elección y segregación debe pasar necesariamente por la indagación de las lógicas de elección de grupos sociales heterogéneos y su interrelación particular, con el contexto más amplio de política en que estas decisiones se llevan a cabo.

En esta línea, este artículo profundiza y contrasta las dinámicas de elección de escuela de familias de clases medias y bajas, buscando comprender los procesos que -desde el lado de la demanda- refuerzan la segregación y estratificación del sistema escolar, en el marco del diseño institucional presente en el país que combina una extensa provisión de educación privada, financiamiento compartido y una selección cuasidesregulada de alumnos por parte de los establecimientos particulares subvencionados. Los resultados provistos por el artículo cuestionan los principios y discursos promulgados por los defensores del school choice en el sistema chileno, entregando importante evidencia en un momento crucial de discusión de una nueva reforma educativa en el país.

\section{ANTECEDENTES Y CONTEXTO. LA FABRICACIÓN DE LA SEGREGACIÓN ESCOLAR MEDIANTE LA ELECCIÓN DE ESCUELA}

\subsection{LA HEGEMONÍA DEL SCHOOL CHOICE Y LA PARTICULARIDAD DE LA POLÍTICA CHILENA}

Durante las últimas décadas, las políticas educativas alrededor del mundo se han visto marcadamente influenciadas por la introducción de elementos neoliberales, como la creación de mercados educativos, la desregulación, la descentralización, el management y la performatividad (Levin, 1998; Ball, 2008; OECD, 2010). Dentro de esta agenda 
neoliberal las políticas de school choice han tenido una posición predominante (Plank \& Sykes, 2003; Forsey, Davies \& Walford, 2008; Ball, 2008; OCDE, 2010; Musset, 2012), siendo tal vez uno de sus aspectos más controversiales.

La expansión del school choice ha descansado en poderosas ideas, remontables a economistas como Hayek o Friedman, quienes establecieron las principales bases del "sentido común" (Levin, 1998) existente frente a estas iniciativas. Así, el discurso propagado en torno al school choice apunta a que mediante el incremento de las posibilidades de elegir y el fomento de la diversidad, los sistemas educativos podrían mejorar su calidad, eficiencia y equidad, esto último al estar otorgando aparentes libres e iguales oportunidades a las familias de hacerse partícipes de estas decisiones y de participar activamente del mercado (Chubb \& Moe, 1990; Henig, 1994; Levin, 1998; Plank \& Sykes, 2003; Forsey et al., 2008; Musset, 2012).

Haciendo eco de las promesas propagadas tras esta política, a partir de la década de 1990, la mayoría de los países de la OCDE han expandido las oportunidades de los padres para elegir una escuela para sus hijos (Musset, 2012). La investigación comparada ha destacado los diversos diseños por los cuales esta política se ha instalado en distintos países (Plank \& Sykes, 2003; Forsey et al., 2008; Mons, 2008; Musset, 2012). Yendo más lejos en esto, Mons estableció una tipología de modelos de elección de escuela en países de la OCDE, compuesta por 4 categorías, a saber: i) el modelo sin elección (Korea o Hong Kong); ii) el modelo limitado de choice, con una asignación de estudiantes por proximidad residencial, con posibilidades de derogación (Francia o Estados Unidos); iii) el modelo de libre elección regulada (Suecia o Dinamarca); y finalmente iv) el modelo de libre elección total (Inglaterra o Nueva Zelanda) (Dupriez, 2010). Acorde a la autora, Chile se ubica dentro de esta última categoría (Mons, 2008).

Las familias chilenas han contado, históricamente, con libertad para elegir una escuela o liceo para sus pupilos, no existiendo en la política educativa del país estrategias de asignación controladas o reguladas de los estudiantes a los establecimientos. No obstante, es claro que la reforma neoliberal impulsada dentro del sistema escolar durante la década de 1980 institucionalizó y expandió la política de school choice bajo el establecimiento de un subsidio a la demanda (o sistema de voucher) y el fomento de la privatización exógena del sistema tendiente a la diversificación de los proveedores educativos. Con todo, Chile aparece como un caso pionero de avance en esta materia (Hsieh \& Urquiola, 2006).

La expansión de las posibilidades de elección parental se introdujo, durante ese periodo, bajo un discurso que subrayó la libertad de la familias a elegir una escuela o liceo en el sector público o privado, con independencia de su lugar de residencia, con la expectativa de que el ejercicio racional de este derecho contribuiría a un aumento de incentivos hacia los establecimientos de mejorar su calidad educativa. La propagación de esta lógica se entrecruzó, asimismo, con una promesa de equidad, que fue entendida como las similares oportunidades de los padres de elegir un establecimiento, con independencia de sus clases sociales. El retorno de la democracia en el país, y más recientemente la Ley General de Educación (MINEDUC, 2009), mantuvo las bases de este sistema, ensalzando el principio de la libre elección bajo la lógica de la calidad y equidad:

El sistema de educación será de naturaleza mixta, incluyendo una de propiedad y administración del Estado y sus órganos, y otra particular, sea esta subvencionada o pagada, asegurándole a los padres y apoderados la libertad de elegir el establecimiento educativo para sus hijos (MINEDUC, 2009: en línea). 
Ahora bien, la política de elección de escuela de Chile se ha impulsado en el país en el marco de un arreglo institucional particular y cuasiinexistente en otras latitudes del mundo, dentro del que destaca: i) la permisión abierta de selección de estudiantes por parte de las escuelas privadas que reciben subvención estatal. Pese a las regulaciones insertas por la LGE en esta ámbito, la evidencia apunta que se trata de una práctica establecida en el sector particular subvencionado (Godoy, Salazar y Treviño, 2014), producto de las debilidades del aparato público por promover el cumplimiento de la normativa; ii) la introducción el año 1993 de La Ley de Financiamiento Compartido, que autorizó a los colegios particulares subvencionados a solicitar un cobro mensual por alumno matriculado adicional a la subvención fiscal, aumentando el aporte de las familias a la educación. Esta normativa se legitimó bajo argumentos basados en la necesidad de entregarle mayor control a las familias de la calidad del servicio educativo ofrecido por las escuelas y captar recursos de quienes no están en situación de pobreza para destinarlos a los que más necesitan (García-Huidobro, 2007); iii) finalmente, a partir de 1995 los resultados SIMCE a nivel de establecimiento escolar se comienzan a difundir públicamente, esperándose que la calidad operara como criterio central de toma de decisión por parte de las familias. De ese tiempo a esta parte, las pruebas se han intensificado, ampliando las materias y cursos sujetos a la evaluación de aprendizaje estandarizada, manteniéndose el discurso orientado al fortalecimiento de la articulación entre elección, información y calidad:

Esta Agencia va a establecer parámetros objetivos de evaluación de los establecimientos educacionales, a través de la prueba Simce, que va a aumentar en frecuencia y que va a cubrir nuevos campos, como el idioma inglés, las tecnologías de la información y la educación física. (...) Todo esto va a ser debida y oportunamente informado a los padres, porque nosotros creemos que la principal responsabilidad en la educación de los hijos recae en sus padres. Y, por tanto, para que ellos puedan ejercer esa responsabilidad, requieren tener información veraz, oportuna y completa, que les permita tomar decisiones informadas y más libres (Piñera, 2011: en línea).

En suma, los discursos promulgados por los defensores del school choice en Chile, aun cuando se institucionalizan con la reforma de los años 80, se han sostenido en las políticas educativas chilenas durante el periodo democrático posterior, estableciéndose una estrecha vinculación entre elección de escuela, calidad y equidad, bajo los preceptos de decisiones aparentemente libres, equitativamente distribuidas e informadas.

\subsection{ELECCIÓN DE ESCUELAS Y SEGREGACIÓN DE LOS SISTEMAS ESCOLARES: EVIDENCIA INTERNACIONAL}

La expansión de las oportunidades de elección de escuelas de las familias a nivel internacional ha coincidido con un marcado interés de la investigación por revelar la operatividad y efectos que estas políticas tienen en el marco de los sistemas escolares más amplios. En un metaanálisis en países de la OCDE, Musset (2012) plantea que los beneficios teóricos [discursivos] de la introducción de mecanismos de mercado no se identifican empíricamente, y que la política provee oportunidades únicamente para algunos padres aventajados, dejando en una posición desigual a las familias de mayor vulnerabilidad.

En un nivel más profundo y desde una perspectiva sociológica, el comportamiento de los actores, en particular de las familias ante la elección de escuela, ha sido tema 
importante en la investigación de la tradición inglesa y francesa sobre el tema. Distintos estudios han concluido así un estrecho vínculo entre el capital social, cultural y económico de los padres y sus elecciones educativas, revelándose la posición privilegiada de las clases medias en este escenario. Dentro de estos, la elección de las familias de clase media se ha catalogado como una práctica e ideología exclusionaria (Van Zanten, 2003), en la que tiene una primacía la composición socioeconómica de las escuelas y la búsqueda de un grupo de pares homogéneo en esta dimensión para los hijos. Generalmente estas decisiones están mediadas por una concepción negativa de los estudiantes de menor nivel socioeconómico, que suponen tener un background académico, familiar y disciplinario perjudicial para el proceso educativo de sus propios hijos (Jellison, 2002; Ball, 2003; Van Zanten, 2003). Estos hallazgos son cruciales para entender las características de la elección de este tipo de familias expuestos por la literatura internacional: las familias de la clase media, tomando ventaja de su posición dentro de la estructura social, sus mayores oportunidades y mayor nivel de información, tienden a i) migrar del sector público hacia el privado, que usualmente cuenta con procesos selectivos del alumnado, o bien ii) salir de las áreas locales de asignación, moviéndose a otros barrios con mejores escuelas públicas desde sus visiones. Como Ball ha planteado:

La toma de decisión para estos padres es un tema de dibujar y redibujar líneas de demarcación, en términos de lo que es aceptable, lo que es necesario, lo que está bajo interés de sus hijos y lo que no. Estos padres crean o protegen las escuelas exclusivas y bajo ello, están generando instituciones que reproducen las clases sociales (Ball, 2003: 83, traducción libre).

En oposición a lo anterior, los estudios enfocados en la elección de las familias de clases sociales bajas han destacado la complejidad de un proceso que se experimenta como dominado por el miedo y usualmente mediado por las propias experiencias negativas de los padres a propósito de sus fracasos en el sistema escolar (Reay \& Ball, 1997). Bajo este escenario, además, las familias más vulnerables tienden a relegarse a las escuelas locales, por sus recursos limitados para pagar la matrícula de las privadas, afrontar los costos de transporte que implica, o por su menor nivel de información y conocimiento acerca de las escuelas (Reay \& Ball, 1997; Broccolichi \& Van Zanten, 2000; Van Zanten \& Ball, 2002). También en esta línea, la literatura apunta a que las familias de estos estratos desarrollan procesos de autoexclusión de escuelas con distinta composición social o étnica a la propia, bajo el temor de sufrir prácticas de discriminación. Siguiendo a Reay \& Ball, "los padres de raza negra deben realizar elecciones más difíciles que sus pares: la elección está inmersa en una realidad de discriminación y dominación" (1997: 91, traducción libre).

A partir de lo anterior, parece existir un consenso de que la contraposición entre las dinámicas y prácticas de elección de escuela de las familias de clase media y baja, y la preponderancia de lógicas de distinción por un lado y de exclusión (y autoexclusión) por el otro, empujan a los sistemas escolares hacia la segregación socioeconómica, o en palabras de Van Zanten \& Ball (2002), impulsan la fabricación de la segregación. El consenso establecido, sin embargo, es sensible hacia el que la estrecha vinculación entre lógicas de elección, clase social y segregación se ven determinadas también por los marcos institucionales y políticas más amplias de los sistemas educativos. Así como da cuenta Ball, "distintos regímenes de política ofrecen distintos tipos de posibilidades 
y ventajas para las clases medias y se vinculan a diversos tipos de contingencia" (Ball, 2003: 56, traducción libre).

\subsection{PROBLEMA: SEGREGACIÓN, CLASES SOCIALES Y ELECCIÓN DE ESCUELA EN EL SISTEMA} ESCOLAR CHILENO

Siguiendo evidencia desarrollada solo más recientemente en Chile, el nivel de segregación y estratificación socioeconómica del sistema educativo es alto (Valenzuela, 2008; Valenzuela, Bellei \& De los Ríos, 2014) y, de hecho, desde una perspectiva comparada el país exhibe uno de los niveles más alarmantes en este ámbito en comparación a los sistemas escolares que participaron en la prueba PISA 2006 (Dupriez, 2010). Con todo, los estudiantes están desigualmente distribuidos según los tipos de escuelas, siendo la clase media principalmente educada en el sistema particular subvencionado y los alumnos más vulnerables en las escuelas públicas (García-Huidobro, 2007).

La investigación centrada en establecer las causas de los indicadores críticos en este tema ha estado esencialmente enfocada en una mirada hacia las condiciones estructurales del país y las normativas que rigen el sistema educativo. De esta forma, elementos como la segregación residencial, el financiamiento compartido y la selección de estudiantes por parte de los establecimientos han sido erigidos concluyentemente como barreras institucionales que favorecen una creciente segregación y segmentación socioeconómica del sistema escolar y no una mejora en su calidad ni un cierre en las brechas de resultados según el estrato social del estudiantado (Bellei, 2007; García-Huidobro, 2007; Valenzuela, 2008; Contreras et al., 2010). En este contexto, el estudio acerca del comportamiento de las familias, las dinámicas y prácticas de elección, y su incidencia en la segregación y estratificación del sistema escolar chileno solo ha comenzado a emerger con fuerza los últimos años dentro del campo de investigación del país, primero desde un enfoque cuantitativo (Elacqua y Fábrega, 2004; Gallego \& Hernando, 2009; Chumacero, Gómez y Paredes, 2011), y con posterioridad desde metodologías cualitativas y etnográficas (Raczynski, Salinas, De la Fuente, Hernández y Lattz, 2010; Raczynski, Hernández, Kegevic y Rocco, 2011; Gubbins, 2013; Córdoba, 2014). En general, la evidencia disponible ha tendido a describir las características de los procesos de elección, particularmente desde las experiencias de las clases bajas, como dan cuenta las dos más recientes publicaciones en esta materia (Gubbins, 2013; Cordoba, 2014).

Tomando en consideración los antecedentes antes planteados, y bajo el supuesto de la íntima relación entre elección, clase social, segregación y diseño institucional del sistema escolar, este artículo se propone ahondar en las dinámicas de elección de escuela y las motivaciones y justificaciones subyacentes del proceso desde los discursos y prácticas de las clases medias y bajas del país. Solo a partir del estudio de la contraposición de ambas dinámicas es posible avanzar hacia una comprensión más profunda respecto de la fabricación de la segregación y estratificación social por parte de las familias en el sistema escolar chileno.

\section{MÉTODO}

Este artículo se apoya en los resultados de dos investigaciones financiadas por el Fondo de Investigación y Desarrollo (FONIDE) del Ministerio de Educación en el 2008 y 2010: 
F310827/2008 "Hacia una estrategia de validación de la educación pública-municipal: imaginarios, valoraciones y demandas de las familias" (Raczynski et al., 2010) y F511066/2010 "El paso de la enseñanza básica a la media en estratos bajos: Un reto a la igualdad de oportunidades educativas" (Raczynski et al., 2011).

Aunque con objetivos principales distintos, ambos estudios incorporaron dentro de sus temáticas la exploración de los procesos de elección de escuelas y liceos, y fueron conducidos a partir de metodologías cualitativas incluyendo la realización de entrevistas individuales y grupales a apoderados del sistema escolar.

De manera más específica, en el primer estudio se llevaron a cabo 12 entrevistas individuales y seis grupales a apoderados de clase media y baja, de las comunas de Puente Alto, Coquimbo y Osorno. Se aseguró en este caso la conducción de entrevistas a igual número de apoderados del sector municipal y particular subvencionado, y de la enseñanza básica y media.

Tabla 1. Entrevistas conducidas en el estudio "Hacia una estrategia de validación de la educación pública municipal: Imaginarios, valoraciones y demandas de las familias"

\begin{tabular}{|l|l|r|}
\hline \multirow{2}{*}{ Entrevistas individuales } & & $\mathbf{N}^{\mathbf{0}}$ \\
\cline { 2 - 3 } & Apoderados GSE media & 12 \\
\cline { 2 - 3 } Entrevistas grupales & Apoderados GSE baja & 12 \\
\cline { 2 - 3 } & Apoderados GSE media & 6 \\
\cline { 2 - 3 } & Apoderados GSE baja & 6 \\
\hline
\end{tabular}

En el segundo estudio se realizaron 34 entrevistas individuales y 11 grupales a apoderados de clase baja, de las comunas de Temuco, Cholchol y Puerto Saavedra de la IX Región, asegurando, como en el caso anterior, diversidad en términos de dependencia administrativa, de niveles de enseñanza y de zona urbana/rural del establecimiento escolar.

Tabla 2. Entrevistas conducidas en el estudio "El paso de la enseñanza básica a la media en estratos bajos: Un reto a la igualdad de oportunidades educativas"

\begin{tabular}{|l|c|}
\hline & $\mathbf{N}^{\mathbf{0}}$ \\
\hline Entrevistas individuales a apoderados de clase baja & 34 \\
\hline & \\
\hline Entrevistas grupales a apoderados de clase baja & 11 \\
\hline & \\
\hline
\end{tabular}

Todas las entrevistas fueron transcritas y se transfirieron a una matriz de vaciado que incorporó las principales dimensiones claves de análisis. La revisión del material

La definición del estrato social del apoderado se realizó a partir de la clasificación de grupo socioeconómico de cada establecimiento escolar levantada por el Ministerio de Educación. 
en ambos estudios se hizo caso por caso y transversalmente por tema, para finalmente analizar semejanzas y diferencias según las diversas distinciones presentes en la selección de la muestra de apoderados, como el nivel de enseñanza, dependencia administrativa del establecimiento, comuna, zona urbana/rural y nivel socioeconómico de la familia,, según la investigación respectiva. Para los propósitos de este artículo, la reflexión se centra únicamente en las distinciones referidas al estrato socioeconómico.

\section{RESULTADOS}

\subsection{LOS PROCESOS DE ELECCIÓN DE ESCUELA: CUESTIONANDO LA ELECCIÓN INFORMADA Y RACIONAL}

La indagación de los procesos de elección de escuela de las familias chilenas pone en tela de juicio la pretensión instalada en la política educativa respecto del peso de SIMCE como orientador de las decisiones en esta materia y la importante limitación que el concepto hegemónico de calidad manejado por los policy makers como sinónimo de resultados de aprendizajes tiene para las familias al momento de realizar sus elecciones.

En primer lugar, las familias de clase media y baja tienen un concepto de calidad más amplio que el que maneja la política educativa, prevaleciendo en ambos grupos una definición multidimensional que incluye distintas dimensiones, como otros estudios y encuestas han postulado (Córdoba, 2006; CIDE, 2006). Dentro de estas se combinan aspectos relacionados a la disciplina, orden y convivencia en el establecimiento, la relación familia-escuela, la preocupación que directores y docentes expresan cotidianamente por sus estudiantes, el aprendizaje que logran los alumnos, la formación valórica que entrega el establecimiento y, en menor medida, la mantención de la infraestructura y disponibilidad de equipamiento escolar. Si bien en varios casos el que un colegio entregue aprendizajes o una buena enseñanza es un elemento mencionado por los apoderados, estos no son siempre prioritarios.

En segundo lugar, las familias manejan poca información objetiva de las escuelas. De este modo, las entrevistas llevadas a cabo con familias de estratos socioeconómicos bajos y medios apuntan a que la mayoría conoce en términos generales la prueba SIMCE, pero pocos tienen una idea acertada respecto de si el colegio tiene un resultado óptimo, regular o deficiente; estos no conocen el puntaje específico del colegio en que se educa(n) su(s) hijo(s) y solo se refieren a sus resultados en términos generales (Gubbins, 2013; Cordoba, 2014). Así, lejos de orientar sus decisiones basadas en el SIMCE, la evidencia da cuenta más bien de la existencia de una notable predominancia de las fuentes primarias y de las redes sociales como medios de información principales, de manera coherente con la literatura internacional en este respecto (Ball \& Vincent, 1998). La información que transita por estas redes, generalmente, es de carácter vago y respecto de una diversidad de ámbitos de los establecimientos, como la disciplina, costos y aprendizajes. Además de lo anterior, las familias tienden a recoger información de las escuelas de forma directa, mediante la observación del comportamiento de los niños/as o jóvenes a la salida del colegio, en que se mira especialmente el lenguaje, la presentación, las conductas y el trato que los estudiantes entregan a sus compañeros y a los mayores, así como si hay o no presencia de adultos y si estos ejercen control sobre las conductas de los estudiantes. 
Los resultados entregados hasta acá cuestionan de forma notoria los principios de una elección informada y racional promovidos por la política educativa en el país, relevando procesos de elección guiados por fuentes de información más amplias y más creíbles a los ojos de las familias, al mismo tiempo que por preocupaciones atinentes a las escuelas y liceos que van más allá que los meros resultados de aprendizaje logrados por los estudiantes. De esta forma, el potencial del modelo instalado en Chile que ha pretendido la existencia de decisiones basadas en los resultados de aprendizajes de los estudiantes (SIMCE) y la calidad definida en esos términos como principio orientador y regulador de la oferta educativa se ve poderosamente limitado, tal como ha planteado Corvalán (2013) respecto de la permanencia de las familias en escuelas de bajo desempeño que sugieren.

Más allá de estas características transversales a los estratos sociales, las siguientes secciones avanzan hacia la comprensión del proceso de elección de escuela de clases medias y bajas.

\subsection{EL PROCESO DE ELECCIÓN PARA LA CLASE MEDIA: LA ELECCIÓN COMO ESTRATEGIA DE DISTINCIÓN}

Los discursos de las familias de clase media revelan la enorme presencia de un proceso de elección configurado bajo una dinámica de distinción y cierre social, que es consistente con la investigación internacional (Ball, 2003; Van Zanten, 2003; Jellison, 2002). Así, aspectos como la importancia de entornos escolares socialmente homogéneos, la valoración de la selección y el cobro de matrícula, y la preponderante mirada negativa hacia educación municipal gratuita, aparecen como criterios dominantes tras sus elecciones en materia educativa.

Desentrañando los elementos antes mencionados, a lo largo de los relatos de las familias de clase media acerca de sus procesos de elección de escuela se vislumbra, en primer lugar, la otorgación de un peso preponderante a lo que ellas revelan como el "tipo de alumno" de un establecimiento, evitando así aquellos colegios que presentan una composición del alumnado indeseable para sus hijos, dentro del que se alude a estudiantes con problemas conductuales, disciplinarios y de familias despreocupadas o sin aspiraciones, o afectados por problemáticas como la drogadicción, alcoholismo, la delincuencia. La consistente evidencia revelada en esta línea indica, así, la existencia de razones "ocultas" o que generalmente reciben baja mención en estudios cuantitativos o encuestas que han buscado identificar las principales razones de la elección (Elacqua y Fábrega, 2004; CEP, 2011). El rechazo al contacto de sus hijos con este "tipo de alumno" -perteneciente dentro de sus representaciones a los estratos vulnerables- se justifica en los discursos familiares bajo la idea de que estos tendrían hábitos sociales y conductas perjudiciales para sus pupilos siguiendo una lógica de contagio social.

Es importante que los niños de nosotros se rodeen con niños que tengan capacidades, estén con buenos niños (apoderado, Puente Alto, particular subvencionado, estrato medio).

Ahí va gente muy pobre y niños bien atrevidos con los profesores. Entonces ahí los otros niños aprenden malas costumbres (apoderado, Coquimbo, particular subvencionado, estrato medio).

En estrecha relación con lo anterior, y bajo la búsqueda de colegios donde los alumnos se encuentran con un medio social similar o mejor al propio, no es sorpresiva la alta valoración existente dentro de este grupo de familias de los colegios que tienen 
procesos de selección del estudiantado, como pruebas de admisión o la exigencia de otros requisitos y métodos tendientes a regular el ingreso de los estudiantes. Estos procesos, que están expandidos usualmente en colegios particulares subvencionados (Godoy et al., 2014), cubren una variedad de aspectos pero se orientan fundamentalmente a indagar en el nivel socioeconómico y cultural de las familias, como se hace evidente en la siguiente descripción de una de las entrevistadas:

¿En qué consiste esa entrevista [en el proceso de admisión]?

La orientadora preguntó dónde vivía, qué tipo de casa, qué relación teníamos con los padres. (...) Si era sólida, básica, si teníamos las necesidades básicas cubiertas, luz, agua, la relación padre e hijo, si yo conversaba con las hijas, si me preocupaba de ir a las reuniones de apoderados, si participaba en el colegio, la relación con otras personas de la familia, si mis hijas participaban en redes sociales, talleres, las habilidades de mis hijas (apoderado, Coquimbo, particular subvencionado, estrato medio).

A la existencia de políticas de admisión exigentes se suma la apreciación positiva por parte de los entrevistados de este estrato, de otras barreras de entrada hacia las escuelas como el cobro o copago de matrícula que opera como un filtro adicional que estaría posibilitando la mantención de la distancia social de sus hijos con alumnos vulnerables.

Yo pago un poquito más y yo sé que no va a ir cualquier niño al colegio (apoderado, Coquimbo, estrato medio).

Dentro de la lógica de las familias, el pago de matrícula aparece, asimismo, como un mecanismo de presión hacia las escuelas para mejorar su calidad, que no existiría en escuelas gratuitas, permitiéndoles adoptar una posición de cliente frente al establecimiento escolar, que es en todos los casos deseada por los apoderados:

[En los colegios pagados] saben que detrás están los apoderados. (...). Son los papás los que exigen y están pagando (apoderado, Puente Alto, estrato medio).

Los mismos apoderados, como tienen que pagarle al colegio, le exigen al colegio y exigen... (apoderado, Coquimbo, estrato medio).

Como es de esperar, la apreciación de la selección y del pago como mecanismos de cierre social que posibilita la construcción de entornos socialmente homogéneos para sus pupilos desplaza a estas familias hacia el sector particular subvencionado con financiamiento compartido, coherente con evidencia de otras latitudes Broccolichi \& Van Zanten, 2000; Jellison, 2002; Veleda, 2007; Van Zanten, 2007). Este proceso se conjuga, adicionalmente, con visiones críticas de la educación pública. En términos transversales, esta visión obedece principalmente a tres discursos fundamentales, recurrentes dentro de las entrevistas efectuadas: i) la educación municipal acoge a estudiantes con atributos negativos o "llega todo tipo de alumnos", lo que impulsa las dos otras referencias negativas, a saber, ii) la educación municipal enfrenta serios problemas de orden, disciplina y convivencia escolar; y iii) ofrece una enseñanza de menor calidad. El que en estos establecimientos no exista la posibilidad de selección de alumnos aparece como el elemento clave que está propiciando e impulsando de manera vigorosa la migración hacia el sector particular y la consecuente evitación de las escuelas públicas: 
Si el niño es desordenado, no lo pueden echar, por ser municipal ellos reciben como las sobras, una cosa así (apoderado, Coquimbo, particular subvencionado, estrato medio).

Es que pienso que no es tanto que los municipales sean malos, sino que la gente que llega a los colegios (apoderado, Puente Alto, particular subvencionado, estrato medio).

Siguiendo la misma idea, la gratuidad de los colegios municipales emerge, desde estas visiones, como un aspecto contraproducente, en la medida que permite la llegada de todo tipo de alumnos y apoderados despreocupados por la educación. Lo anterior, en la perspectiva de las familias, repercute en la ausencia de reglas y disciplina dentro de las escuelas.

[Los colegios municipales] son más económicos, accesibles, no se pagan mensualidades, pero (...) no hay reglas, el niño no va a clases porque la mamá se enfermó, 'ah bueno' le dicen, no existe ese tipo de control, son menos controlados internamente... (apoderado, Coquimbo, particular subvencionado, estrato medio).

La dinámica de distinción y de cierre social que subyace a los procesos y prácticas de elección de escuela de las familias de clase media es fuertemente propulsada, como se puede entrever, por la propia institucionalidad del sistema y política chilena que posibilita selección y cobro de matrícula en el sector particular subvencionado. El sistema de elección de escuela en el marco del diseño de política más amplio instalado en el país se convierte así en un escenario propicio dentro del cual las familias pueden efectivamente desplegar sus estrategias de distinción mediante sus decisiones educativas, haciendo uso de sus ventajas comparativas en la disposición de capital económico, cultural y social.

\subsection{EL PROCESO DE ELECCIÓN DE LA CLASE BAJA: LA ELECCIÓN COMO EXCLUSIÓN}

En oposición a las dinámicas prevalecientes en los procesos de elección de las clases medias del país, las decisiones educativas en este plano en apoderados de clases bajas se enmarcan dentro de una importante lógica de exclusión y frustración, producida por sus menores recursos económicos para participar de la aparente libertad de elección y realizar elecciones deseables para sus hijos.

Siguiendo la idea principal, los procesos de elección de las familias de estratos vulnerables revelan el peso primero y fundamental de las consideraciones prácticas, a saber, distancia y costo, dentro de sus principales razones de elección, evidencia que es consistente con la proporcionada por otras investigaciones conducidas en apoderados de estas características ((Elacqua y Fábrega, 2004; Gubbins, 2013).

Vi ese colegio, lo vi más cerca, no tengo para pagar locomoción, mensualidad y por eso me quedé (apoderado, Coquimbo, estrato bajo).

La consideración de las razones prácticas como principal factor de elección de un establecimiento se experimenta en un escenario en que lo deseable y preferible son colegios de mayor costo o de otra dependencia que, sin embargo, son imposibles de elegir por los límites que sus condiciones económicas les imponen. 
La mayoría de los colegios particulares quedan en el centro de la ciudad entonces eso implica tenerle al niño la locomoción, un furgón y por supuesto la matrícula que también es alta, entonces por eso no se pudo colocar en un colegio particular (apoderado, Osorno, estrato bajo).

[Ese colegio] vale $\$ 80.000$ mensuales. Si yo tuviera esos medios, pongo a mis hijos en colegios particulares. Pero lamentablemente, uno tiene que ser realista (apoderado, Coquimbo, estrato bajo).

Considerando las restricciones que enfrentan, los apoderados de este estrato se ven forzados al repliegue hacia la oferta local, municipal o particular no selectiva, en un proceso que es en algunos casos experimentado con una evidente frustración, también identificada por Gubbins (2013).

Se lo elige [el liceo municipal de Saavedra] porque es el único y no porque sea bueno. Este es un pueblo de bajos recursos. Si uno alega le dicen: qué alega si no paga nada. Yo dejé a mi hijo contra mi voluntad ahí (apoderado, Saavedra, estrato bajo).

Es que yo a veces quisiera a lo mejor otro colegio, pero el sistema económico también falla, porque hay colegios que uno tiene que pagar más y como este es municipal (apoderado, Temuco, estrato bajo).

Al mismo tiempo, las familias perciben y son sensibles a las imágenes negativas que pesan en los colegios a los que asisten sus hijos, dando cuenta de las dinámicas de segregación a las que se ven expuestos.

[Conversando con otras madres] te dicen es que tengo a mi hija en el XX [establecimiento particular subvencionado] y se le suben los humos y tú te quedas callada porque estás en uno municipal, entonces los que están en los particulares conversan entre ello (apoderado, Osorno, estrato bajo).

Ante este escenario, los apoderados buscan, al menos discursivamente, marginarse de la visión propagada de las escuelas a las que asisten sus hijos, destacando cualidades positivas de su establecimiento e intentando borrar aquellas imágenes difundidas entre los sectores medios sobre la realidad educativa que se vive en sus colegios.

El XX [municipal] dicen que son pelusas, como que la gente lo ve como que son cabros drogadictos, delincuentes y cuando tú ves y trabajas acá es como que no es tan así, porque a mí los chicos nunca me han faltado el respeto y yo les digo a la gente que no diga eso (apoderado, Osorno, estrato bajo).

En esa instancia [al momento de escoger colegio para enseñanza media], como uno ignora lo de los particulares, uno como que creía que en los particulares no había desorden, pero con el paso de los años uno va dándose cuenta que en todos los liceos pasan cosas... si uno ve en la micro chicas del XX groseras, insolentes, entonces yo estaba conforme con el YY [municipal] que es un liceo bueno y hay profesionales con trabajo que salen bien de ahí (apoderado, Osorno, estrato bajo).

En definitiva, la dinámica de exclusión y frustración que rodea los procesos de elección de las familias más vulnerables del país se ve configurada por las evidentes restricciones y límites que su propia posición dentro de la estructura social les impone 
para la toma de decisiones. Estos resultados ponen en entredicho los supuestos en los que opera la política de school choice, mediante la constatación más bien de un escenario en el que las clases bajas corren con desventaja.

\section{CONCLUSIÓN Y DISCUSIÓN}

La evidencia acumulada de un tiempo a esta parte en Chile relativas a los procesos de elección de escuela desde enfoques cualitativos, a la que este artículo aporta, ha apuntado considerablemente hacia un cuestionamiento de los principios y discursos orientadores de la política del school choice implementada en el país. Así, la supuesta elección informada, racional, libre e igualmente distribuida entre estratos sociales tiende a derrumbarse al entrar con profundidad en las experiencias, prácticas y los propios discursos de las familias sobre su toma de decisión en este plano.

La profundización provista por este trabajo en las dinámicas de elección de clases medias y bajas chilenas da cuenta de un proceso guiado por una estrategia de distinción de la primera y, de una concomitante y opuesta lógica de exclusión de los estratos vulnerables, que repercute y refuerza la segregación y estratificación del sistema educativo. El estrecho vínculo entre clases sociales, elecciones y segregación socioeconómica del sistema escolar del país no es neutro al diseño institucional de la política instalada en Chile. Así, la permisión de selección de alumnos por parte de las escuelas, el financiamiento compartido y la lógica de competencia entre el sector municipal y particular subvencionado aparecen como escenarios propicios para el despliegue de las dinámicas identificadas que tienden fuertemente hacia una reproducción de la estructura y cierre social.

Esta evidencia cobra particular relevancia en el marco de los anuncios de una nueva reforma educacional en el sistema escolar chileno. Como el artículo sugiere, a partir de su reflexión, la disminución de los indicadores críticos a nivel de la segregación y estratificación social del sistema y la promoción de la mixtura social dentro de los establecimientos escolares requiere el avance hacia un cambio profundo del actual diseño institucional, orientado hacia la derogación del financiamiento compartido y de la selección por parte de las escuelas y liceos, que debe ir acompañado de un fortalecimiento de la deteriorada imagen de la educación pública. El progreso en esta línea, finalmente, debe enmarcarse en un proceso cultural más amplio y profundo que borre prejuicios y encauce conductas favorables a la diversidad social, la no discriminación e inclusión social. En esa perspectiva, la propia educación debiese hacerse cargo de promover estas conductas tanto en la formación inicial y en servicio de los docentes como dentro de los objetivos claves del currículum nacional.

\section{REFERENCIAS BIBLIOGRÁFICAS}

Ball, S. (2008). The education debate. Bristol: The Policy Press.

. (2003). Class strategies and the education market: The middle classes and social advantage. Oxon: Routledge Falmer.

Ball, S. \& Vincent, C. (1998). I heard it on the grapevine: Hot knowledge and school choice. British Journal of Sociology of Education, vol. 10 (3), 377-400. 
Bellei, C. (2007). Expansión de la educación privada y mejoramiento de la educación en Chile. Evaluación a partir de la evidencia. Pensamiento Educativo, vol. 40 (1), 285-311.

Broccolichi, S. \& Van Zanten, A. (2000). School competition and pupil flight in the urban periphery. Journal of Education Policy, vol. 15 (1), 51-60.

CEP (2011). Estudio nacional de opinión pública $N^{o}$ 4. Santiago: Centro de Estudios Públicos. Recuperado el 14 de mayo de 2014 desde http://www.cepchile.cl/dms/archivo_4844_2963/encuestaCEP_junio-julio2011_completa.pdf

Chubb, J. \& Moe, T. (1990). Politics, markets and America's schools. Washington D.C.: The Brookings Institution.

Chumacero, R., Gómez, D. \& Paredes, R. (2011). I would walk 500 miles (if it paid): Vouchers and school choice in Chile. Economics of Education Review, vol. 30 (5), 1103-1114.

CIDE (2006). VI Encuesta a actores del sistema educativo. Santiago: Universidad Alberto Hurtado. Recuperado el 14 de mayo de 2014 desde http://biblioteca.uahurtado.cl/ujah/Reduc/pdf/ $\mathrm{pdf} / \mathrm{mfn} 250 . \mathrm{pdf}$

Contreras, D., Sepúlveda, P. \& Bustos, S. (2010). When schools are the ones that choose: The effects of screening in Chile. Social Science Quarterly, vol. 91 (5), 1349-1368.

Córdoba, C. (2014). La elección de escuela en sectores pobres: Resultados de un estudio cualitativo. Psicoperspectivas. Individuo y Sociedad, vol. 13 (1), 68-87.

(2006). Elección de escuela en sectores pobres de Chile. Simposio PENS/EDU-1:

Políticas Educacionales y Gestión de Sistemas Escolares. Madrid, España. Recuperado el 13 de mayo 2014 desde http://www.reseau-amerique-latine.fr/ceisal-bruxelles/PENS-EDU/PENS-EDU1-CORDOBA.pdf

Corvalán, J. (2013). El concepto de buena escuela en apoderados cuyos hijos asisten a escuelas de bajo rendimiento en Chile: Un análisis antropológico-estructural. Papeles de Trabajo. Centro de Estudios Interdisciplinarios en Etnolingüística y Antropología Socio-Cultural, (26), 62-87.

Dupriez, V. (2010). Séparer pour réussir? Les modalités de groupement des élevés. Paris: UNESCO.

Elacqua, G. y Fábrega, R. (2004). El consumidor de la educación: El actor olvidado de la libre elección de escuelas en Chile. Recuperado el 18 de mayo de 2014 desde http://www.preal.org/ Archivos/Programas/Seminarios\%20y\%20Talleres/Accountability\%20Educacional/Documentos\%20 de\%20Referencia/ASOCFILE.pdf

Forsey, M., Davies, S. \& Walford, G. (2008). The globalization of school choice? Oxford: Symposium Books.

Gallego, F. \& Hernando, A. (2009). School choice in Chile: Looking at the demand side. Retrieved May 14, 2014 from http://www.economia.puc.cl/docs/dt_356.pdf

García-Huidobro, J. E. (2007). Desigualdad educativa y segmentación del sistema escolar. Consideraciones a partir del caso chileno. Pensamiento Educativo, vol. 40 (1), 65-85.

Godoy, F., Salazar, F. y Treviño, E. (2014). Prácticas de selección en el sistema escolar chileno: Requisitos de postulación y vacíos legales. Informes para la Política Educativa, (1), 1-9.

Gubbins, V. (2013). La experiencia subjetiva del proceso de elección de establecimiento educacional en apoderados de escuelas municipales de la Región Metropolitana. Estudios Pedagógicos, vol. 39 (2), 165-177.

Henig, J. (1994). Rethinking school choice: Limits of the market metaphor. New Jersey: Princeton University Press.

Hsieh, C. T. \& Urquiola, M. (2006). The effects of generalized school choice on achievement and stratification: Evidence from Chile's school voucher program. Journal of Public Economics, vol. 90, 1477-1503.

Jellison, J. (2002). Buying homes, buying schools: School choice and the social construction of school quality. Harvard Educational Review, vol. 72 (2), 177-205.

Levin, B. (1998). An epidemic of education policy: (What) can we learn from each other? Comparative Education, vol. 34 (2), 131-141. 
MINEDUC (2009). Ley General de Educación. Recuperado el 14 de mayo 2014 desde http:// www.mineduc.cl/usuarios/convivencia_escolar/doc/201103050142570.Ley_N_20370_Ley_General_ de_Educacion.pdf

Mons, N. (2008). Les nouvelles politiques éducatives. La France fait-elle les bons choix? Paris: Presses Universitaires de France.

Musset, P. (2012). School choice and equity: Current policies in OECD countries and a literature review. OECD Education Working Papers, (66). Retrieved May 17, 2014 from http://www. oecd-ilibrary.org/education/school-choice-and-equity_5k9fq23507vc-en

OECD. (2010). Markets in education: An analytical review of empirical research in market mechanisms in education. OECD Education Working Paper, (52). Retrieved May 17, 2014 from http:// search.oecd.org/officialdocuments/displaydocumentpdf/?cote=EDU/WKP(2010)15\&docLanguage=En

Piñera, S. (2011). Palabras de S. E. el Presidente de la República, Sebastián Piñera, al promulgar la Ley de Aseguramiento de la Calidad de la Educación. Recuperado el 14 de mayo de 2014 desde http://www.prensapresidencia.cl/discurso.aspx? codigo $=7152$

Plank, D. \& Sykes, G. (2003). Choosing choice: School choice in international perspective. New York: Teachers College Press.

Raczynski, D., Hernández, M., Kegevic, L. y Rocco, R. (2011). El paso de la enseñanza básica a la media en estratos bajos: Un reto a la igualdad de oportunidades educativas. Recuperado el 14 de mayo de 2014 desde http://www.comunidadescolar.cl/documentacion/FONIDE/Informe $\% 20$ Final-Dagmar\%20Raczynski-F511083.pdf

Salinas, D., De la Fuente, L., Hernández, M. y Lattz, M. (2010). Hacia una estrategia de validación de la educación pública-municipal: Imaginarios, valoraciones y demandas de las familias. Recuperado el 14 de mayo de 2014 desde http://www.facso.uchile.cl/psicologia/epe/_documentos/GT_cultura_escolar_politica_educativa/recursos\%20bibliograficos/articulos\%20relacionados/ razcynskietal(2010)haciaunaestraegiadecalidaddelaeducacionpublicamunicipal.pdf

Reay, D. \& Ball, S. (1997). Spoilt for choice: The working classes and educational markets. Oxford Review of Education, vol. 23 (1), 89-101.

Valenzuela, J. P., Bellei, C. y De los Ríos, D. (2014). Socioeconomic school segregation in a market-oriented educational system. The case of Chile. Journal of Education Policy, vol. 29 (2), 217-241.

(2008). Evolución de la segregación socioeconómica de los estudiantes chilenos y su relación con el financiamiento compartido. Recuperado el 14 de mayo de 2014 desde http://www. facso.uchile.cl/psicologia/epe/_documentos/GT_cultura_escolar_politica_educativa/recursos $\% 20$ bibliograficos/articulos\%20relacionados/valenzuelabelledelosros(2009)segregacionyficom.pdf

Van Zanten, A. (2007). Reflexividad y elección de la escuela por los padres de la clase media en Francia. Revista de Antropología Social, vol. 16, 245-278.

(2003). Middle-class parents and social mix in French urban schools: Reproduction and transformation of class relations in education. International Studies in Sociology of Education, vol. 13 (2), 107-124.

et Ball, S. (2002). La construction des ségrégations scolaires en France et en Angleterre. Dans Ministère de l'Éducation (Ed.), La discrimination positive en France et dans le monde (pp. 33-53). Paris: CNDP. Consulté le 14 mai 2014 à partir de http://www2.cndp.fr/archivage/ valid/43322/43322-8263-9409.pdf

Veleda, C. (2007). Entre querer y poder. Las clases medias y la elección de la escuela en el conurbano bonaerense. En M. Narodowski y M. Gómez Schettini (Eds.), Escuelas y familias. Problemas de diversidad cultural y justicia social (pp. 127-172). Buenos Aires: Prometeo Libros. 
